\title{
Diferentes niveles de energía metabolizable y aminoácidos azufrados en dietas para gallinas Bovans blancas
}

\section{Different levels of metabolizable energy and sulfur amino acids in diets for white Bovans hens}

\author{
María de los Ángeles Tepox Péreza , Benjamín Fuente Martíneza, Tomás Jínez Méndeza, \\ Ernesto Ávila Gonzáleza
}

\begin{abstract}
RESUMEN
Con el objeto de evaluar el comportamiento productivo de la gallina Bovans blanca de primer ciclo alimentadas con dietas con niveles diferentes de energía metabolizable (EM) y de metionina+cistina digestible (AAD), para lograr disminuir el porcentaje de huevo pequeño $(<42 \mathrm{~g})$, al inicio de la producción se utilizaron 384 gallinas de 19 semanas de edad. Se usó un diseño completamente al azar, en un arreglo factorial de $4 \times 2$, donde el primer factor fueron los niveles de EM $(2700,2800,2900,3000 \mathrm{Kcal} / \mathrm{kg})$ y el segundo factor fue la inclusión de diferentes porcentajes de AAD $(0.45,0.61)$. Cada tratamiento, contó con cuatro repeticiones de 12 gallinas cada uno. Durante ocho semanas se llevaron registros semanales, del porcentaje de postura, peso promedio del huevo, consumo de alimento; masa de huevo/ave/día y conversión alimenticia; además, se clasificó el huevo de acuerdo al peso. Los resultados obtenidos, no mostraron respuesta a los factores de estudio ni a la interacción entre nivel de EM y AAD, para ninguna de las variables productivas. Se encontró un efecto sobre el consumo de alimento del nivel de energía y aminoácidos azufrados $(P<0.05)$. Se disminuyó el porcentaje de huevo pequeño en los niveles más altos de EM de 2900 y $3000 \mathrm{Kcal} / \mathrm{Kg}(29.6$ y $30.4 \%$ respectivamente). Se incrementó $(P<0.05)$ el porcentaje de huevo grande en $3 \%$ al usar dietas con el nivel de AAD de $0.61 \%$.
\end{abstract}

PAlabras ClaVE: Peso de huevo, Energía metabolizable, Acido linoleico, Aminoácidos azufrados digestibles.

\begin{abstract}
In order to evaluate the performance early egg production in the white Bovans hen fed diets with different levels of metabolizable energy (ME) and digestible methionine + cystine (AAD), to reduce the percentage of small eggs $(<42 \mathrm{~g})$, three hundred and eighty four (384) hens 19-wk of age were used in a completely randomized design in a factorial arrangement of $4 \times 2$ where the first factor were the levels of ME $(2700,2800,2900,3000 \mathrm{Kcal} / \mathrm{kg})$ and the second factor was the inclusion of two levels of ADD (0.45, 0.61). Each treatment had four replications of 12 hens each. Egg production, egg weight, feed intake, egg mass and feed conversion were weekly measured for $8 \mathrm{wk}$, moreover, were classified according to egg weight. The results showed no effect for main factors and level of interaction between ME and the AAD for productive traits; an effect was found for feed intake and energy levels of sulfur amino acid levels $(P<0.05)$. Higher levels of 2900 and $3000 \mathrm{ME} \mathrm{kcal} / \mathrm{kg}(29.6$ and $30.4 \%$ respectively) reduced the percentage of small eggs. Percentage of large eggs was increased in $3 \%$ by using diets with $0.61 \%$ AAD level $(P<0.05)$.
\end{abstract}

KEY WORDS: Weight egg, Metabolizable energy, Sulfur amino acids.

\section{INTRODUCCIÓN}

Al inicio del ciclo de postura las gallinas ponen huevos pequeños o "pee wee", y la cantidad de estos disminuye a medida que aumenta la edad de

\section{INTRODUCTION}

At the beginning, of the laying cycle, hens lay small eggs or "peewee", and the amount of these eggs decreases with increasing age of the hen; it is about

Recibido el 4 de mayo de 2011. Aceptado el 14 de septiembre de 2011.

a Centro de Enseñanza, Investigación y Extensión en Producción Avícola (CEIEPAv) De la Facultad de Medicina Veterinaria y Zootecnia de la Universidad Nacional Autónoma de México. angeles_lvgv6@ hotmail.com. Correspondencia al primer autor. 
la gallina; es alrededor de la semana 40 cuando las ponedoras incrementan el tamaño del huevo a grande, extra grande o jumbo. Al respecto, hay pocas investigaciones encaminadas a mejorar el tamaño del huevo al inicio de la producción, se toman en cuenta factores que hacen variable el peso del huevo; algunos son resultado del manejo y otros tienen una relación genética. Aunque cada línea genética tiene determinado potencial para cierto tamaño de huevo, depende del manejo de la parvada para que las aves muestren una completa expresión de su potencial( ${ }^{(1)}$.

A pocas semanas de alcanzar la madurez sexual y antes de que las gallinas lleguen a su pico de producción, gran parte del número de huevos puestos son de bajo valor económico, debido a su pequeño peso. En el presente trabajo, se estudió una alternativa alimenticia que permita optimizar los parámetros productivos deseados, ya que entre más grande sea el huevo en las primeras semanas de producción de la gallina, el ingreso será mayor.

El peso del huevo es resultado de caracteres genéticos cuantitativos con un alto grado de heredabilidad (55\%), siendo las líneas más pesadas y semi-pesadas las que tienden a producir huevos de mayor peso respecto a las líneas ligeras(2).

Algunas prácticas de manejo, con las cuales se puede modificar y evitar ligeramente el bajo peso del huevo al inicio de la postura y lograr que la mayoría de los huevos cumpla con el estándar de la clasificación comercial usada, son la uniformidad en el peso corporal, edad a la madurez sexual, programa de iluminación y alimentación ${ }^{(2,3-6)}$.

De acuerdo a los programas de alimentación, el consumo de energía al inicio de la producción es un factor importante que influye en el pico de postura y en el peso temprano del huevo, éste puede ser modificado por medio de niveles adecuados de energía metabolizable y aminoácidos incluidos en las $\operatorname{dietas}(7)$.

Las gallinas de postura, son capaces de regular su consumo de alimento incrementándolo conforme la wk 40 when laying hens increase egg size to large, extra large or jumbo. There is little research to increase the size of the egg at the beginning of production, taking into account factors as management and genetics. Although each strain has a certain potential for egg size, to show a complete expression of their potential depends on the handling of the hens ${ }^{(1)}$.

Few weeks before reaching sexual maturity and before the laying hens reach their peak production, much of the laid eggs are of low economic value due to their small weight. In this paper, we studied a food alternative for optimizing production parameters, since the bigger the egg in the first weeks of production, the higher of the income.

Egg weight is a result of quantitative genetic characters with a high degree of heritability (55\%); and heavier and semi-heavy lines tend to produce more heavier eggs with respect to light lines $(2)$.

Management practices that can slightly change and avoid the low egg weight at the start of the laying, and make that most of the eggs meet the standard trade classification used, are uniformity in body weight, age at sexual maturity, lighting and feeding $\operatorname{program}^{(2,3-6)}$.

According to feeding programs, energy intake at the start of production is a decisive factor that influences peak laying and weight of the egg, which can be changed through appropriate levels of metabolizable energy and amino acids included in the $\operatorname{diets}(7)$.

Lying hens are able to regulate their feed intake, increasing it as egg production increases. The amount of feed consumed varies according to age and stage of production and must meet the metabolizable energy (ME) requirements, under ambient and appropriate management conditions ${ }^{(8.9)}$.

The ME is one of the most influential factors on egg weight; inclusion of 1.2 to $1.4 \%$ fats gave better results. Fats or oils of vegetable or animal origin as well as being sources of energy are sources 
producción de huevo aumenta. La cantidad de alimento ingerido varía de acuerdo a la edad y a la etapa de producción, y debe satisfacer el requerimiento de energía metabolizable (EM), esto bajo condiciones ambientales y de manejo adecuadas $(8,9)$.

La EM es uno de los factores con mayor importancia sobre el peso del huevo; al incluir 1.2 a $1.4 \%$ de grasa, se obtiene la mejor respuesta. Las grasas o aceites de origen vegetal o animal, además de ser fuentes de energía son fuentes de ácidos grasos esenciales. Al incorporar aceite en la dieta, el peso del huevo puede aumentar $2 \mathrm{~g}$, esto sólo ocurre cuando se usan grasas no saturadas. Cuando las dietas contienen un nivel de ácido linoleico mayor de $1.5 \%$ en el inicio de la producción, se obtienen mejores pesos de huevo, y niveles de $3 \%$ mejoran en $1 \mathrm{~g}$ el tamaño de huevo, en comparación con las dietas que contienen $1.5 \%(7,9,10)$.

No existe un requerimiento de proteína per se, pero éste debe ser capaz de satisfacer las necesidades de aminoácidos esenciales, tomando en cuenta su digestibilidad. Hay autores que señalan que al aumentar la proteína cruda de la dieta de inicio de las ponedoras de 17 a $22 \%$, el peso del huevo aumenta $(11,12)$.

La metionina debe ser aportada en cantidades importantes, porque el organismo de las aves es incapaz de sintetizar este aminoácido. Al formular dietas a base de sorgo + soya, la metionina es el primer aminoácido limitante. Es posible modificar el peso de huevo en base al consumo de metionina+cistina, con $700 \mathrm{mg} / \mathrm{ave} / \mathrm{día}$ al inicio de la postura con buenos resultados $(7,10)$.

Se ha demostrado un efecto lineal en el peso del huevo al incrementar los niveles de aminoácidos azufrados en la dieta de 0.65 a $0.81 \%$, concluyendo que el peso del huevo se incrementa $0.7 \mathrm{~g}$ por cada $0.05 \%$ de incremento de aminoácidos azufrados en gallinas de 59 semanas de edad(13).

Se reconoce que el contenido de aminoácidos esenciales afecta el consumo de alimento, por lo of essential fatty acids. By incorporating dietary oil (unsaturated fats), egg weight may increase $2 \mathrm{~g}$. When diets contain a linoleic acid level higher than $1.5 \%$ at the start of production, the better the egg weights; and levels of $3 \%$ increase in $1 \mathrm{~g}$ egg size, compared to diets containing $1.5 \%(7,9,10)$.

There is no requirement for protein per se, but it must be able to meet the needs of essential amino acids, taking into account their digestibility. Some authors point out that increasing crude protein in starter diet of laying hens from 17 to $22 \%$, egg weight increases $(11,12)$.

Methionine should be provided in large quantities because the birds' body is incapable of synthesize this amino acid. In diets based on sorghum + soybean, methionine is the first limiting amino acid. Egg weight can be changed with satisfactory results providing $700 \mathrm{mg} / \mathrm{bird} / \mathrm{d}$ of methionine + cystine at the onset of lay $(7,10)$.

It has shown a linear effect on egg weight by increasing levels of sulfur amino acids in the diet from 0.65 to $0.81 \%$, concluding that egg weight increases $0.7 \mathrm{~g}$ by every $0.05 \%$ increase in sulfur amino acids in 59-wk old laying hens(13).

It is recognized that the content of essential amino acids affects the feed intake, so diets deficient in one or more essential amino acid results in a lower feed intake and poor weight gain(7).

\section{MATERIALS AND METHODS}

The research was conducted at the Center for Teaching, Research and Extension in Poultry Production (CEIEPAv), Faculty of Veterinary Medicine, of the National University of Mexico, located at 2,250 m, $19^{\circ} 15^{\prime} \mathrm{N}$ and $99^{\circ} 02^{\prime} 30^{\prime \prime} \mathrm{W}$, humid temperate climate $(\mathrm{Cw})$, January being the coldest month and May the warmest; average temperature of $16{ }^{\circ} \mathrm{C}$ and average annual rainfall of $747 \mathrm{~mm}^{(14)}$. Three hundred and eighty four (384) Bovans white 19-wk old chickens with an average body weight of $1,246 \pm 20.5 \mathrm{~g}$ were housed in 
que dietas deficientes en uno o más aminoácidos esenciales dan como resultado un menor consumo de alimento y una baja ganancia de peso $^{(7)}$.

\section{MATERIALES Y MÉTODOS}

La investigación se realizó en el Centro de Enseñanza, Investigación y Extensión en Producción Avícola (CEIEPAv) de la Facultad de Medicina Veterinaria y Zootecnia, de la Universidad Nacional Autónoma de México, localizado a 2,250 msnm, en $19^{\circ} 18^{\prime} \mathrm{N}, 9^{\circ} 02^{\prime} \mathrm{O}$. Con clima templado cages, in a natural environment, and distributed under a completely randomized design in 32 groups of 12 birds each.

Treatments or experimental diets and calculated analyzes are in Tables 1 and 2; diets with $0.45 \%$ digestible methionine + cystine sugar were added to replacing them with DL-methionine and having the following amino acid level.

Food and water were provided ad libitum throughout the experiment. Treatments consisted

Cuadro 1. Composición de las dietas basales experimentales para gallinas

Table 1. Composition of experimental basal diets for laying hens

\begin{tabular}{lcccr}
\hline & \multicolumn{4}{c}{ ME (Kcal / kg) } \\
\cline { 2 - 5 } Ingredient & 2700 & 2800 & 2900 & 3000 \\
\hline Sorghum & 659.17 & 650.70 & 625.94 & 601.20 \\
Soybean meal & 190.02 & 196.32 & 202.21 & 208.10 \\
Calcium carbonate & 102.54 & 102.50 & 102.44 & 102.40 \\
Wheat bran & 12.47 & 0.00 & 0.00 & 0.00 \\
Calcium phosphate & 11.22 & 11.42 & 11.47 & 11.53 \\
Vegetable oil & 6.00 & 20.60 & 39.6 & 58.58 \\
Salt & 4.40 & 4.40 & 4.41 & 4.41 \\
Vitamin and mineral mixture* & 2.50 & 2.50 & 2.50 & 2.50 \\
L-lisina HCl & 2.22 & 2.10 & 1.96 & 1.81 \\
Mycotoxin binder & 2.00 & 2.00 & 2.00 & 2.00 \\
Vegetable yellow pigment ${ }^{* *}$ & 1.20 & 1.20 & 1.20 & 1.20 \\
Vegetable red pigment*** & 1.00 & 1.00 & 1.00 & 1.00 \\
DL-Methionine & 0.51 & 0.51 & 0.52 & 0.52 \\
Choline chloride 60, \% & 0.50 & 0.50 & 0.50 & 0.50 \\
Bacitracin zinc & 0.30 & 0.30 & 0.30 & 0.30 \\
Antioxidant & 0.10 & 0.10 & 0.10 & 0.10 \\
Phytase ${ }^{* \star \star}$ & 0.10 & 0.10 & 0.10 & 0.10 \\
Sugar & 3.75 & 3.75 & 3.75 & 3.75 \\
Total & 1000 & 1000 & 1000 & 1000 \\
\hline
\end{tabular}

* Vit $A$ 3,833.000 IU, Vit $D_{3} 1,500.00 \mathrm{IU}$, Vit E 13,333.500 mg, Vit $K_{3} 1,333.275 \mathrm{mg}$, Vit $B_{1} 499.560 \mathrm{mg}$, Vit $B_{2}$ $2,000.000 \mathrm{mg}$, Vit $B_{6} 1,000.400 \mathrm{mg}$, Vit $B_{12} 6,670 \mathrm{mg}$, nicotinamide $15,000.00 \mathrm{mg}$, pantothenic acid $3,332.700 \mathrm{mg}$, folic acid $277.600 \mathrm{mg}$, biotine $40.00 \mathrm{mg}$, choline chloride 133,333.200 mg, Cu 3,333.350 mg, Fe 23,333.250 mg, Mn $37,888.820 \mathrm{mg}$, yodo $333.400 \mathrm{mg}$, zinc $26,666.640 \mathrm{mg}$, Se $100.000 \mathrm{mg}$, calcium carbonate $370.000 \mathrm{~g}$, mineral oil 5.000 g, vehicle c.b.p $1.000 \mathrm{~kg}$.

** (avelut): yellow xantophiles $15 \mathrm{~g} / \mathrm{kg}$.

*** (avired in dust): $5 \mathrm{~g} / \mathrm{kg}$, capsicum.

**** phytase of bacterial origin E. coli, phytase IP6, products of Saccharomyces pomde fermentation, wheat flour, calcium propionate, citric acid. 
húmedo $\mathrm{Cw}$, siendo enero el mes más frío y mayo el más caluroso, temperatura promedio anual de $16{ }^{\circ} \mathrm{C}$ y precipitación pluvial anual media de $747 \mathrm{~mm}^{(14)}$. Se utilizaron 384 gallinas Bovans blancas de 19 semanas de edad, con un peso corporal promedio de $1,246 \pm 20.5 \mathrm{~g}$. Las gallinas fueron alojadas en jaulas en una caseta de ambiente natural, y se distribuyeron conforme a un diseño completamente al azar, en 32 grupos de 12 aves cada uno.

Los tratamientos o dietas experimentales y los análisis calculados se muestran en los Cuadros $1 \mathrm{y}$ 2 , a las dietas con $0.45 \%$ de metionina+cistina digestible se les adicionó azúcar para sustituirlas por DL-Metionina y tener el siguiente nivel de aminoácidos.

El alimento y el agua se ofrecieron a libre acceso durante todo el experimento. Los tratamientos consistieron en el empleo de diferentes niveles de EM y metionina+cistina digestible (AAD). Se empleó un diseño completamente al azar con un arreglo factorial de 4 X 2; el primer factor fueron los niveles de EM $(2700,2800,2900,3000 \mathrm{Kcal} /$ $\mathrm{kg}$ ) y el segundo factor 0.45 y $0.61 \%$ de AAD. Cada tratamiento, contó con cuatro repeticiones de 12 gallinas cada uno

Se llevaron registros semanales durante ocho semanas, de porcentaje de postura, peso promedio de huevo, consumo de alimento; se calculó la masa de huevo por ave por día y la conversión alimenticia; además, de la semana 19 a la 26 se clasificó el huevo de acuerdo a la medida estadounidense ${ }^{(15)}$. Al final del estudio a los datos de las variables obtenidas, se les realizó un análisis conforme al diseño experimental empleado, usando el paquete estadístico Jmp 8.0(16). Se realizaron regresiones lineales y cuadráticas para consumo de alimento, consumo de EM con los diferentes niveles de energía metabolizable en la dieta y consumo de energía por ave con peso del huevo.

\section{RESULTADOS Y DISCUSIÓN}

En el Cuadro 3, se muestran los resultados promedio de 8 semanas de experimentación; no se
Cuadro 2. Análisis calculado de las dietas experimentales empleadas (\%)

Table 2. Chemical composition of the diets (\%)

\begin{tabular}{lcccc}
\hline & \multicolumn{4}{c}{ ME (Kcal/kg) } \\
& 2700 & 2800 & 2900 & 3000 \\
\hline Crude protein & 15.18 & 15.24 & 15.32 & 15.40 \\
Digestible met+cis & 0.453 & 0.453 & 0.454 & 0.455 \\
Digestible lysine & 0.803 & 0.803 & 0.802 & 0.801 \\
Digestible threonine & 0.477 & 0.481 & 0.484 & 0.488 \\
Available phosphorus & 0.440 & 0.440 & 0.440 & 0.440 \\
Calcium & 4.000 & 4.000 & 4.000 & 4.000 \\
Linoleic acid & 1.151 & 1.744 & 2.666 & 3.574 \\
Ether extract & 2.540 & 3.960 & 5.690 & 8.320 \\
\hline
\end{tabular}

$\mathrm{ME}=$ metabolizable energy.

on the use of different levels of $\mathrm{ME}$ and digestible methionine+cystine (DAA). A completely randomized design with a factorial arrangement of 4 X 2 was used, the first factor were the levels of ME $(2700,2800,2900,3000 \mathrm{Kcal} / \mathrm{kg}$ ) and the second factor two levels of 0.45 and $0.61 \%$ DAA. Each treatment had four replicates of 12 hens each.

Weekly for $8 \mathrm{wk}$, laying percentage, egg average weight, and feed intake were recorded; the mass of eggs per bird per day and feed conversion were also calculated; from wk 19 to 26 eggs were classified according to the USA measurements(15). At the end of study data variables obtained, were analyzed according to the experimental design, using the Jmp 8.0 statistical package(16).

Linear and quadratic regressions for feed intake and MS consumption with different metabolizable energy levels in the diet and energy consumption per bird with egg weight were conducted.

\section{RESULTS AND DISCUSSION}

Table 3 shows the average results of 8 -wk experiment; there were no effects of the factors studied and the level of interaction between ME and DAA $(P>0.05)$ for laying percentage, egg weight, egg mass and feed conversion, neither linear 
encontraron efectos de los factores estudiados ni interacción entre el nivel de EM y AAD $(P>0.05)$ para las variables porcentaje de postura, peso de huevo, masa del mismo y conversión alimentaria, ni tendencia lineal y cuadrática para peso de huevo con respecto al consumo de EM y los diferentes niveles de AAD. Alguna literatura señala que no hay un efecto sobre el peso del huevo al incrementar la energía de 2,600 a 3,000 Kcal/kg, debido a la disminución del consumo de otros nutrientes ${ }^{(18-20)}$. Otros estudios señalan que con niveles de aminoácidos azufrados entre 0.574 y $0.81 \%$ de AAD no se afecta el porcentaje de postura ni el peso del huevo(21,22).

Para consumo de alimento, hubo respuesta a niveles de EM y AAD $(P<0.05)$. El consumo de alimento (96.1 g) disminuyó en el nivel de $3000 \mathrm{Kcal}$ con respecto a las dietas con 2700, 2800 y $2900 \mathrm{Kcal}$ (99.1, 99.7 y $99.1 \mathrm{~g}$ respectivamente). Al realizar la regresión para consumo de alimento con los diferentes niveles de energía metabolizable en la dieta (consumo de alimento= 124.5-0.009*nivel de EM en la dieta), los resultados mostraron una $\mathrm{r}^{2}=$ $12.9 \%(P<0.01)$, valor muy bajo, por lo que no se consideró. Al respecto varios autores(20,23-27) mencionan que la gallina es capaz de ajustar su consumo de alimento de acuerdo a sus necesidades, los cambios del medio ambiente y dependiendo de la cantidad de energía de la dieta; en general, a mayor EM el consumo de alimento disminuye. Los resultados de esta prueba corroboraron esta aseveración sólo en dietas de $3000 \mathrm{Kcal}$, las cuales tuvieron un $3 \%$ menos consumo de alimento con respecto a las otras dietas. Otros investigadores encontraron una disminución de hasta un $10 \%$ en dietas con $3000 \mathrm{Kcal}$ con respecto a otras de 2750 Kcal; se ha señalado que al incrementar $22 \mathrm{kcal}$ en la dieta el consumo de alimento disminuye en $1 \%$, y que las gallinas blancas son menos sensibles a los cambios de energía de la dieta, en comparación con gallinas rojas $(27,28)$. También se valoró el consumo de EM/Kcal/ave/día con los diferentes niveles de energía metabolizable en la dieta, y no se encontró efecto lineal ni cuadrático para esta variable $(P>0.05)$; siendo mayor significativamente
Cuadro 3. Resultados promedio de parámetros productivos en gallinas de postura alimentadas con diferentes niveles de energía y aminoácidos azufrados digestibles

Table 3. Average results of productive parameters in laying hens fed with different levels of energy and digestible sulfur amino acids (DAA)

\begin{tabular}{|c|c|c|c|}
\hline \multirow[b]{2}{*}{ EM Kcal/kg } & \multicolumn{2}{|c|}{ DAA $\%$} & \multirow[b]{2}{*}{ Mean \pm SE } \\
\hline & 0.45 & 0.61 & \\
\hline \multicolumn{4}{|c|}{ Egg production (\%) } \\
\hline 2700 & $68.8 \pm 2.59$ & $65.8 \pm 2.59$ & $67.3 \pm 1.83 a$ \\
\hline 2800 & $66.0 \pm 2.59$ & $69.0 \pm 2.59$ & $67.5 \pm 1.83^{\mathrm{a}}$ \\
\hline 2900 & $66.2 \pm 2.59$ & $62.4 \pm 2.59$ & $64.3 \pm 1.83^{a}$ \\
\hline 3000 & $69.4 \pm 2.59$ & $67.5 \pm 2.59$ & $68.4 \pm 1.83^{a}$ \\
\hline Average & $67.6 \pm 1.29 a$ & $66.2 \pm 1.29 a$ & \\
\hline \multicolumn{4}{|c|}{ Egg weight (g) } \\
\hline 2700 & $46.8 \pm 1.14$ & $49.1 \pm 1.14$ & $48.0 \pm 0.80^{a}$ \\
\hline 2800 & $48.4 \pm 1.14$ & $49.0 \pm 1.14$ & $48.7 \pm 0.80^{a}$ \\
\hline 2900 & $48.2 \pm 1.14$ & $46.6 \pm 1.14$ & $47.5 \pm 0.80^{a}$ \\
\hline 3000 & $47.2 \pm 1.14$ & $49.8 \pm 1.14$ & $48.5 \pm 0.80^{a}$ \\
\hline Average & $47.7 \pm 0.57 a$ & $48.6 \pm 0.57 a$ & \\
\hline \multicolumn{4}{|c|}{ Egg mass/hen/d (g) } \\
\hline 2700 & $34.4 \pm 1.39$ & $33.7 \pm 1.39$ & $34.0 \pm 0.98 \mathrm{a}$ \\
\hline 2800 & $33.4 \pm 1.39$ & $35.4 \pm 1.39$ & $34.4 \pm 0.98 \mathrm{a}$ \\
\hline 2900 & $34.5 \pm 1.39$ & $32.2 \pm 1.39$ & $33.4 \pm 0.98^{a}$ \\
\hline 3000 & $35.0 \pm 1.39$ & $35.2 \pm 1.39$ & $35.1 \pm 0.98^{a}$ \\
\hline Average & $34.3 \pm 0.69 a$ & $34.1 \pm 0.69 a$ & \\
\hline \multicolumn{4}{|c|}{ Feed intake/d (g) } \\
\hline 2700 & $101.0 \pm 1.02$ & $97.3 \pm 1.02$ & $99.1 \pm 0.72^{a}$ \\
\hline 2800 & $100.6 \pm 1.02$ & $98.8 \pm 1.02$ & $99.7 \pm 0.72^{a}$ \\
\hline 2900 & $100.6 \pm 1.02$ & $97.5 \pm 1.02$ & $99.1 \pm 0.72^{a}$ \\
\hline 3000 & $98.1 \pm 1.02$ & $94.0 \pm 1.02$ & $96.1 \pm 0.72^{b}$ \\
\hline Average & $100.1 \pm 0.51^{a}$ & $97.0 \pm 0.51^{b}$ & \\
\hline \multicolumn{4}{|c|}{ Consumption of ME (Kcal/hen/d) } \\
\hline 2700 & $272 \pm 2.3$ & $262 \pm 2.3$ & $267 \pm 1.68 \mathrm{c}$ \\
\hline 2800 & $281 \pm 2.3$ & $276 \pm 2.3$ & $279 \pm 1.68^{b}$ \\
\hline 2900 & $291 \pm 2.3$ & $283 \pm 2.3$ & $287 \pm 1.68 \mathrm{a}$ \\
\hline 3000 & $294 \pm 2.3$ & $282 \pm 2.3$ & $288 \pm 1.68 \mathrm{a}$ \\
\hline Average & $285 \pm 1.19 a$ & $276 \pm 1.19 b$ & \\
\hline \multicolumn{4}{|c|}{ Feed conversion $(\mathrm{kg}: \mathrm{kg})$} \\
\hline 2700 & $3.4 \pm 0.42$ & $3.7 \pm 0.42$ & $3.6 \pm 0.30^{a}$ \\
\hline 2800 & $3.7 \pm 0.42$ & $3.8 \pm 0.42$ & $3.7 \pm 0.30^{a}$ \\
\hline 2900 & $3.4 \pm 0.42$ & $4.7 \pm 0.42$ & $4.1 \pm 0.30^{a}$ \\
\hline 3000 & $3.26 \pm 0.42$ & $3.1 \pm 0.42$ & $3.2 \pm 0.30^{a}$ \\
\hline Average & $3.45 \pm 0.21^{a}$ & $3.85 \pm 0.21^{a}$ & \\
\hline
\end{tabular}

$\mathrm{SE}=$ standard error.

ab Values with different superscript differ $(P<0.05)$. 
en las aves que consumieron dietas con 2900 y $3000 \mathrm{Kcal}$ en la dieta, en donde el consumo de energía/día/ave fue de 287 y 288 Kcal respectivamente (Cuadro 3).

Hubo una disminución del $3 \%$ en el consumo de alimento $(P<0.05)$, cuando se usaron dietas con el nivel más alto de AAD (0.61\%). Algunos aminoácidos regulan el consumo de alimento por medio de los receptores que estimulan el apetito; una marginalidad de los aminoácidos plasmáticos activan o inhiben el centro del apetito, lo que provoca una disminución en el consumo, y por lo tanto también en nutrientes(29-31).

El menor consumo de AAD fue de $720 \mathrm{mg}$ ave/ día; el NRC ${ }^{(32)}$ recomienda en promedio para esa etapa de producción un consumo de $580 \mathrm{mg} /$ día, por lo que se cubrieron los requerimientos de estos aminoácidos. En relación a la conversión alimenticia, el manual de la estirpe ${ }^{(15)}$ recomienda en promedio de la semana 19 a la 25 una conversión de 5.41, aunque no proporciona los valores de la semana 18; se calculó el valor faltante y se obtuvo una conversión de 8.54 en promedio, conversión menor que la obtenida en este trabajo (3.65).

De acuerdo a la clasificación americana del huevo en porcentaje, no se encontró respuesta a los diferentes niveles de energía $(P>0.05)$, para el huevo jumbo, extra grande, grande, mediano y bajo (Cuadro 4). Se logró disminuir el porcentaje de huevo pequeño con los niveles más altos de EM $(P<0.05)$, con respecto a las dietas que tenían el nivel más bajo; probablemente debido al incremento del consumo del ácido linoleico, ya que se ha señalado que niveles superiores al $2 \%$ mejoran el peso del huevo; otros indican que hay una estimulación directa de los estrógenos por los ácidos grasos de la dieta(33-35).

El aumento en el peso del huevo también está asociado con el aumento del peso de la albúmina, por una respuesta directa al contenido de aminoácidos(36), por lo que probablemente se obtuvo un efecto significativo en el porcentaje de huevo grande, el cual se incrementó en $3 \%$; así
Cuadro 4. Producción de huevo en gallinas Bovans blancas de la semana 19 a 25 (en porcentaje) de acuerdo a la clasificación tipo americana

Table 4. Egg production in white Bovans laying hens of the wk 19-25 (in percentage) according to the American type classification

\begin{tabular}{|c|c|c|c|}
\hline \multirow[b]{2}{*}{ ME (Kcal/kg) } & \multicolumn{2}{|c|}{ DAA $\%$} & \multirow[b]{2}{*}{ Mean $\pm S E$} \\
\hline & 0.45 & 0.61 & \\
\hline \multicolumn{4}{|c|}{ Jumbo } \\
\hline 2700 & $1.2 \pm 0.51$ & $1.3 \pm 0.51$ & $1.2 \pm 0.36$ \\
\hline 2800 & $1.2 \pm 0.51$ & $2.0 \pm 0.51$ & $1.6 \pm 0.36 \mathrm{a}$ \\
\hline 2900 & $2.3 \pm 0.51$ & $0.9 \pm 0.51$ & $1.6 \pm 0.36$ \\
\hline 3000 & $0.9 \pm 0.51$ & $0.9 \pm 0.51$ & $0.9 \pm 0.36 \mathrm{a}$ \\
\hline Average & $1.4 \pm 0.25 \mathrm{a}$ & $1.3 \pm 0.25 \mathrm{a}$ & \\
\hline \multicolumn{4}{|c|}{ Extra large } \\
\hline 2700 & $1.4 \pm 0.59$ & $1.4 \pm 0.59$ & $1.40 \pm 0.41$ \\
\hline 2800 & $0.5 \pm 0.59$ & $0.7 \pm 0.59$ & $0.60 \pm 0.41$ \\
\hline 2900 & $2.2 \pm 0.59$ & $1.3 \pm 0.59$ & $1.73 \pm 0.41$ \\
\hline 3000 & $1.6 \pm 0.59$ & $0.7 \pm 0.59$ & $1.17 \pm 0.41$ \\
\hline Average & $1.4 \pm 0.29 a$ & $1.0 \pm 0.29 \mathrm{a}$ & \\
\hline \multicolumn{4}{|c|}{ Large } \\
\hline 2700 & $4.3 \pm 1.77$ & $5.96 \pm 1.77$ & $5.1 \pm 1.25$ \\
\hline 2800 & $3.8 \pm 1.77$ & $6.49 \pm 1.77$ & $5.2 \pm 1.25 a$ \\
\hline 2900 & $5.9 \pm 1.77$ & $9.20 \pm 1.77$ & $7.6 \pm 1.25$ \\
\hline 3000 & $6.1 \pm 1.77$ & $10.40 \pm 1.77$ & $8.3 \pm 1.25$ \\
\hline Average & $5.0 \pm 0.88 a$ & $8.00 \pm 0.88$ b & \\
\hline \multicolumn{4}{|c|}{ Medium } \\
\hline 2700 & $36.7 \pm 2.72$ & $42.0 \pm 2.72$ & $39.3 \pm 1.92$ \\
\hline 2800 & $41.7 \pm 2.72$ & $41.7 \pm 2.72$ & $43.8 \pm 1.92$ \\
\hline 2900 & $44.5 \pm 2.72$ & $48.1 \pm 2.72$ & $46.3 \pm 1.92$ \\
\hline 3000 & $45.4 \pm 2.72$ & $46.7 \pm 2.72$ & $46.0 \pm 1.92$ \\
\hline Average & $42.1 \pm 1.36 \mathrm{a}$ & $45.6 \pm 1.36 \mathrm{a}$ & \\
\hline \multicolumn{4}{|c|}{ Small } \\
\hline 2700 & $40.6 \pm 3.01$ & $35.3 \pm 3.01$ & $37.9 \pm 2.13 \mathrm{a}$ \\
\hline 2800 & $37.6 \pm 3.01$ & $29.6 \pm 3.01$ & $33.6 \pm 2.13 \mathrm{ab}$ \\
\hline 2900 & $31.0 \pm 3.01$ & $30.0 \pm 3.01$ & $30.4 \pm 2.13 \mathrm{ab}$ \\
\hline 3000 & $31.5 \pm 3.01$ & $27.6 \pm 3.01$ & $29.6 \pm 2.13$ \\
\hline Average & $35.2 \pm 1.50 \mathrm{a}$ & $30.6 \pm 1.50 \mathrm{~b}$ & \\
\hline \multicolumn{4}{|c|}{ Low } \\
\hline 2700 & $15.8 \pm 1.99$ & $14.1 \pm 1.99$ & $14.9 \pm 1.40$ \\
\hline 2800 & $15.1 \pm 1.99$ & $15.2 \pm 1.99$ & $15.1 \pm 1.40$ \\
\hline 2900 & $14.1 \pm 1.99$ & $10.5 \pm 1.99$ & $12.3 \pm 1.40$ \\
\hline 3000 & $14.4 \pm 1.99$ & $13.6 \pm 1.99$ & $14.0 \pm 1.40$ \\
\hline Average & $14.8 \pm 0.99 \mathrm{a}$ & $13.3 \pm 0.99 \mathrm{a}$ & \\
\hline
\end{tabular}

$\mathrm{SE}=$ standard error.

ab Values with different superscript differ $(P<0.05)$.

$\mathrm{DAA}=$ digestible amino acids. 
como reducción del huevo pequeño en $13 \%$ $(P<0.05)$ con el nivel de AAD $(0.61 \%)$.

\section{CONCLUSIONES E IMPLICACIONES}

Se puede concluir que al incrementar los niveles de 2700 a $3000 \mathrm{Kcal} / \mathrm{kg}$ de EM y AAD en dietas para gallinas desde que éstas rompen postura hasta que alcanzan el pico de producción, no se afectan los parámetros productivos. Con niveles de 2900 y $3000 \mathrm{Kcal} / \mathrm{kg}$ de EM en la dieta de gallinas Bovans blancas de 18 a 25 semanas de edad se disminuye el porcentaje de huevo pequeño. $\mathrm{Al}$ emplear niveles de aminoácidos azufrados de $0.61 \%$, se incrementa el porcentaje de huevo grande y disminuye el porcentaje de huevo pequeño.

\section{LITERATURA CITADA}

1. North MO, Bell DD. Manual de producción avícola.3 $3^{\mathrm{a}}$ ed. México DF: El Manual Moderno; 1993.

2. Fuente MB. Factores que afectan el tamaño del huevo. Zootecnia y manejo de las aves productoras de huevo comercial. Sistema de Universidad Abierta. México DF [en prensa].

3. Elliot AM. Application of new concepts in pullet and layer nutrition. Congreso CLANA; Colegio Latinoamericano de Nutrición Animal A.C. Cancún, Quintana Roo, México. 2008:199-220.

4. Quintana LJA. Crianza de gallinas ponedoras. En: diplomado en producción avícola. Modulo I. Zootecnia avícola. Escamilla GM, Parra HMA, Vargas AM, editores. México UNAM-FMVZ: 1999:51-57.

5. Nilipour HA. Factores que afectan el tamaño de huevo. Industria Avícola 1995:8-10.

6. ISA. Joly P. Factors that influence egg weight: how to change it to meet market requirements? A Hendrix Genetics Company. Saint Brieuc, France. 2007.

7. Cuca GM, Ávila GE, Pro MA. Alimentación de las aves. Universidad Autónoma Chapingo. Dirección de Patronato Universitario. Departamento de Zootecnia. 2008.

8. Martínez AC. Avances en nutrición de gallina de postura. Congreso CLANA; Colegio Latinoamericano de Nutrición Animal A.C. Cancún, Quintana Roo, México. 2008:221-224.

9. Leeson S, Summers JD. Commercial poultry nutrition. $3^{\text {rd }}$ ed. Canada: University Books; 2005.

10. López CC. Optimización de la nutrición de ponedoras en nuestros días. Congreso CLANA; Colegio Latinoamericano de Nutrición Animal A.C. Cancún, Quintana Roo, México. 2008:193-197. and quadratic trend for egg weight in relation to ME intake and different DAA levels. Some literature indicates that there is no effect on egg weight by increasing the energy of 2600 to $3000 \mathrm{kcal} / \mathrm{kg}$ due to decreased intake of other nutrients(18-20). Other studies indicate that sulfur amino acid levels between 0.574 and $0.81 \%$ does not affect egg laying rate and egg weight $(21,22)$.

$\mathrm{ME}$ and DAA levels modified feed intake response $(P<0.05)$. Feed intake $(96.1 \mathrm{~g})$ decreased in the level of $3000 \mathrm{Kcal} v s$ diets with 2700, 2800 and $2900 \mathrm{kcal}$ (99.1, 99.7 and $99.1 \mathrm{~g}$ respectively). Performing the regression for feed intake with different metabolizable energy levels in the diet (feed intake 124.5-0.009* ME level in the diet), the results showed a $\mathrm{r}^{2}=12.9 \%(P<0.01)$ value exceeding low, so it was not considered. Several authors $(19,20,23-27)$ mention that the hen adjust their feed intake according to their needs, environmental changes and depend on the amount of energy from the diet; in general at higher ME feed intake decreases. The results of this test corroborated this statement only in diets with $3000 \mathrm{kcal}$, which had $3 \%$ less feed intake in relation to the other diets. Other researchers found a decrease of up to $10 \%$ in diets with $3000 \mathrm{kcal}$ with respect to $2750 \mathrm{Kcal}$, and noted that increasing $22 \mathrm{kcal}$ in the diet reduced feed intake by $1 \%$ and that white chickens are less sensitive to changes in dietary energy, vs red hens $(27,28)$. It was also evaluated the use of ME/ $\mathrm{kcal} / \mathrm{bird} / \mathrm{d}$ with different metabolizable energy levels in the diet, and found no linear or quadratic effect for this variable $(P>0.05)$, being significantly higher in birds fed diets with 2900 and $3000 \mathrm{kcal}$ in the diet, with 287 and $288 \mathrm{kcal} /$ respectively (Table 3).

Feed intake decreased $3 \%(P<0.05)$ with the highest DAA diets level $(0.61 \%)$. Some amino acids regulate feed intake via receptors which stimulate the appetite, a plasma margin amino acid activate or inhibits the appetite center, causing a decrease in consumption, and therefore, also in the nutrients(29-31).

The lower consumption of DAA was $720 \mathrm{mg}$ hen/d; the $\mathrm{NRC}^{(32)}$ recommended on average for that stage 
11. De Blas C, Mateos GG. Nutrición y alimentación de gallinas ponedoras. Madrid: Ediciones Mundi-Prensa; 1991.

12. Keshavarz K, Nakajima S. The effect of dietary manipulations of energy, protein and fat during the growing and laying periods on early egg and egg components. Poult Sci 1995;74:50-61.

13. Calderón VM, Jensen LS. The requirement for sulfur amino acid by laying hens as influenced by the protein concentration. Poult Sci 1990;69:934-944.

14. Instituto Nacional de Estadística y Geografía. Tlahuac: Cuaderno de información básica delegacional. México, DF: INEGI, 1992.

15. Manual Bovans White 2003.

16. Jmp8.0. Copyright ${ }^{\circledR}$ 2008. SAS Institute inc.

17. Totsuka K, Okazaki Y, Yamamoto A, Koide K, Watanabe E, Toyomizu $\mathrm{M}$ et al. Effect of dietary crude protein and metabolizable energy levels on the performance of laying hens. Jpn Poult Sci 1993;30:1-15.

18. Keshavarz K. The effect of light, floor space, and energy and protein levels during the growing period on body weight and early egg size. Poult Sci 1998:77:1266-1279.

19. Wu G, Bryant M, Voitle RA, Roland DA. Effect of dietary energy on performance and egg composition of bovans white and dekalb white hens during phase I. Poult Sci 2005;84:16101615.

20. Wu G, Gunawardana P, Bryant MM, Roland DA. Influence of dietary energy and antibiotic on performance, egg solids, and egg quality in bovans white and delkab white hens. J App Poult Res 2008;17:323-330.

21. Zollitsch W, Jevne C, Leske K, Coon C. Utilización de aminoácidos cristalinos en las dietas de gallinas ponedoras. Ciclo de conferencias sobre aminoácidos sintéticos. México DF. Fermex A.C. 1993.

22. Ahmad HA, Roland DA. Effect of method of feeding and feed formulation on performance and profitability of laying hens: an econometric approach. J Appl Poult Sci Res;2003:12:291298.

23. Waldroup PW, Hellwig HM. Methionine and total sulfur amino acid requirements influenced by stage of production. J Appl Poult Res 1995;4:283-292.

24. Fuente MB, Gonzales ER, Ávila GE. Comportamiento productivo de gallinas ligeras con dietas con diferente concentración energética. Congreso Nacional de AMENA; Puerto Vallarta, Jalisco, México. Asociación Mexicana de Especialistas en Nutrición Animal A.C. 1997.

25. Harms RH, Russell GB. The influence of methionine on commercial laying hens. J Appl Poult Res 1998;7:45-52.

26. Harms RH, Russell GB, Sloan DR. Performance of four strains of commercial layers with major changes in dietary energy. $\mathrm{J}$ Appl Poult Res 2000;9:535-541.

27. Harms RH, Russell GB. Performance of commercial laying hens when fed diets with various sources of energy. J Appl Poult Res 2004;13:365-369.

28. Yousefi M, Shivazad M, Sohrabi-Haghdoost I. Effects of reducing dietary methionine, linoleic acid, choline and increasing energy on performance and eggshell quality in aged laying hens. European Poultry Conference; Verona, Italia. World Poultry Sci Assoc. 2006. of production an intake of $580 \mathrm{mg} / \mathrm{d}$, to cover the amino acid requirements. In relation to feed conversion, the Strain manual ${ }^{(15)}$ recommends for wk 19 to 25 an average conversion of 5.41 , but gives no values at wk 18; by calculation of the missing value, 8.54 was obtained, lower conversion than that obtained in this work (3.65).

According to the American classification as a percentage, there was no response to the different energy levels $(P>0.05)$ for the jumbo, extra large, large, medium and low size eggs (Table 4). It is possible to reduce the percentage of small egg with the highest ME levels $(P<0.05)$ with respect to the diet that had the lowest level, probably due to increased intake of linoleic acid, since levels above $2 \%$ improved egg weight. Others suggest a direct stimulation of estrogen by dietary fatty acids (33-35).

The increase in egg weight it is also associated with increased albumin weight, a direct response to the amino acid content ${ }^{(36)}$; probably the DAA level of $0.61 \%$ had a significant effect in the percentage of large eggs, which increased in $3 \%$, and reduction of $13 \%$ in small eggs $(P<0.05)$.

\section{CONCLUSIONS AND IMPLICATIONS}

It can be concluded that increasing the levels from 2700 to $3000 \mathrm{Kcal} / \mathrm{kg} \mathrm{ME}$ and DAA in diets for laying hens since they start laying until they reach the peak production, productivity parameters are not affected. Levels of 2900 and $3000 \mathrm{Kcal} / \mathrm{kg} \mathrm{ME}$ in the diet of 18 to 25 wk-old white Bovans laying hens decreases the percentage of small eggs. When using sulfur amino acid levels of $0.61 \%$, the percentage of large eggs increases and decreases the percentage of small eggs.

End of english version

29. Narvaez.-Solarte W. Rostagno HS, Soares PR, Silva MA, Uribe.F. Nutritional requirements in methionine + cyistine for white-egg laying hens during the first cycle of production. Int J Poult Sci 2005;4(12):965-968. 
30. Peters JC, Harper AE. Adaptation of rats to diets containing different levels of protein: effect on food intake, plasma and brain amino acid concentration and brain neurotransmitter metabolism. J Nutrition 1985;115;382-398.

31. Harper AE. Amino acid imbalances, toxicities and antagonisms. Nutr Rev 1965;14:225-227.

32. NRC. National Research Council. Nutrients requirements of poultry; 9th Revised ed. Washington, DC: National Academy Press; 1994

33. Scragg RH, Logan NB, Geddes N. Response of weight to the inclusion of various fats in layers diets. Br Poult Sci 1987;28:15-21.
34. Whitehead CC, Bowman AS, Griffin HD. Regulation of plasma oestrogen by dietary fats in the laying hen: Relationship with egg weight. Br Poult Sci 1993;34:999-1010.

35. Novak C, Yakout H, Scheideler S. The combined effect of dietary lysine and total sulfur amino acid level on egg production parameters and egg components in dekalb delta laying hens. Poult Sci 2004;83:977-984.

36. Chan- Colli M, Carvajal M, Segura J, Sarmiento L, Santos R. Effect of dietary energy and sulphur amino acid level on egg production traits in the tropics. J Anim Vet Adv 2007;6(10):1209-1213. 